\title{
SOME CHARACTERIZATIONS OF TOTALLY NONPOSITIVE (TOTALLY NEGATIVE) MATRICES*
}

\author{
RAFAEL CANTÓ ${ }^{\dagger}$, BEATRIZ RICARTE ${ }^{\dagger}$, AND ANA M. URBANO ${ }^{\dagger}$
}

\begin{abstract}
An $n \times m$ real matrix $A$ is said to be totally nonpositive (totally negative) if every minor is nonpositive (negative). In this paper, we study characterizations of these classes of matrices by minors, by their full rank factorization and by their thin $Q R$ factorization.
\end{abstract}

Key words. Totally nonpositive and totally negative matrices, Full rank factorization, Thin QR factorization.

AMS subject classifications. 65F40, 15A15, 15A23.

1. Introduction. A matrix $A \in \mathbb{R}^{n \times m}$ is called totally nonpositive (totally negative) if all its minors are nonpositive (negative) and abbreviated as t.n.p. (t.n.). For square t.n. matrices, spectral properties and $L D U$ factorization are analyzed in [9], and a characterization in terms of the parameters of the Neville elimination is obtained in [12]. For nonsingular t.n.p. matrices, an $L D U$ factorization and some properties are presented in [3]. In addition, characterizations by minors for rectangular t.n.p. and t.n. matrices are obtained in [5] taking into account their full rank factorization in echelon form.

The t.n.p. (t.n.) matrices can be considered an extension of the partially nonpositive matrices, p.n.p. (partially negative matrices, p.n.), that is, matrices such that all its principals minors are nonpositive (negative). A p.n. matrix is called $N$-matrix in economic models [2]. Also, the p.n. matrices arise in conjunction with Lemke's algorithm for solving linear and convex quadratic programming problems [16, 17].

On the other hand, the totally positive matrices, TP (strictly totally positive matrices, STP), that is, matrices such that all its minors are nonnegative (positive) have a wide variety of applications in approximation theory, numerical mathematics, statistics, economics and others fields. Note that TP and STP matrices have been studied extensively $[1,2,6,7,8,10,11,12,13,15]$, but there exist few papers on t.n.p. and t.n. matrices $[3,5,9]$. A relation between the TP and t.n.p. matrices is given

\footnotetext{
* Received by the editors June 3, 2009. Accepted for publication April 19, 2010. Handling Editor: Joao Filipe Queiro.

${ }^{\dagger}$ Institut de Matemàtica Multidisciplinar, Universidad Politécnica de Valencia, 46071 Valencia, Spain (rcanto@mat.upv.es, bearibe@mat.upv.es, amurbano@mat.upv.es). Supported by the Spanish DGI grant MTM2007-64477 and by the UPV under its research program.
} 
by the following result: "the product of two t.n.p. matrices is a TP matrix". But the question: "Can any $n \times n$ TP matrix be written as the product of two t.n. matrices?" is an open problem, which can be found in the initial paper for t.n. matrices [9].

To solve this open problem, we study and characterize the t.n. matrices. We extend the well-known results on TP matrices for t.n. and t.n.p. matrices. Hence, the main goal of this paper is to derive characterizations of rectangular t.n.p. (t.n.) matrices by minors and in terms of their thin $Q R$ factorization.

2. Notation and definitions. Following the notation of [1], given $k, n \in \mathbb{N}$, $1 \leq k \leq n$, the subcollection of all such sequences that consist of consecutive integers is denoted by $\mathcal{Q}_{k, n}$. When the natural numbers are consecutive the sequence is denoted by $\mathcal{Q}_{k, n}^{0}$. If $A \in \mathbb{R}^{n \times m}, \alpha \in \mathcal{Q}_{k, n}$ and $\beta \in \mathcal{Q}_{k, m}$ with $k=1,2, \ldots, \min \{n, m\}, A[\alpha \mid \beta]$ denotes the $k \times k$ submatrix of $A$ lying in rows $\alpha$ and columns $\beta$. The principal submatrix $A[\gamma \mid \gamma]$ is abbreviated as $A[\gamma]$, where $\gamma \in \mathcal{Q}_{k, \min \{n, m\}}$. Therefore, $A \in$ $\mathbb{R}^{n \times m}$ is t.n.p. (t.n.) if $\operatorname{det} A[\alpha \mid \beta] \leq 0(<0)$, for all $\alpha \in \mathcal{Q}_{k, n}$ and $\beta \in \mathcal{Q}_{k, m}$ with $k=1,2 \ldots, \min \{n, m\}$.

We recall that a matrix is an upper echelon matrix if it satisfies the following conditions:

1. The first nonzero entry in each row is called leading entry for that nonzero row.

2. Each leading entry is to the right of all first leading entry in the row above it.

3. All zero rows are at the bottom.

If, in addition, the matrix satisfies the following condition is called upper reduced echelon matrix

4. Each leading entry is the only nonzero entry in its column.

A matrix is a lower (reduced) echelon matrix if its transpose is an upper (reduced) echelon matrix. Moreover, if each leading entry is equal to 1 , we add the adjective unit to these definitions.

Given a matrix $A \in \mathbb{R}^{n \times m}$ with $\operatorname{rank}(A)=r$, a decomposition $A=F G$ is called full rank factorization of $A$ if $F \in \mathbb{R}^{n \times r}, G \in \mathbb{R}^{r \times m}$ and $\operatorname{rank}(F)=\operatorname{rank}(G)=r$. It is known that the full rank factorization of any nonzero matrix is not unique but if the matrix $G$ is a unit upper reduced echelon matrix then this factorization is unique [4].

Recall that a characterization for square TP and STP matrices by their $Q R$ factorization is obtained in [11]. For the rectangular case, these characterizations are 
extended in [6] by using their thin $Q R$ factorization.

From now on, we suppose that $A=\left(a_{i j}\right)$ has not zero rows and zero columns. In addition, as it can be proved applying [3, Proposition 3.2] and [5, Theorem 9], if $A$ is a t.n.p. matrix with $a_{11}<0$ then $a_{i j}<0$ for $i=1,2, \ldots, n$ and $j=1,2, \ldots, m$, except for $a_{n m} \leq 0$.

3. Characterization of rectangular t.n.p. matrices by minors. The main goal of this section is to obtain characterizations of rectangular t.n.p. matrices which reduce the number of minors to be checked to deduce if a matrix is t.n.p.

Proposition 3.1. Let $A=\left(a_{i j}\right) \in \mathbb{R}^{n \times r}$ be a matrix with $\operatorname{rank}(A)=r, a_{n r} \leq 0$ and the remaining entries less than zero. Consider the nonsingular matrix $A_{1} \in \mathbb{R}^{r \times r}$ formed by the first $r$ linearly independent rows of $A$. Then, $A$ is t.n.p. if and only if for each $k=1,2, \ldots, r$, the following inequalities hold

$$
\begin{aligned}
\operatorname{det} A[\alpha \mid 1,2, \ldots, k] & \leq 0, \quad \text { for all } \alpha \in \mathcal{Q}_{k, n} \\
\operatorname{det} A_{1}[1,2, \ldots, k \mid \beta] & \leq 0, \quad \text { for all } \beta \in \mathcal{Q}_{k, r} \\
\operatorname{det} A_{1}[1,2, \ldots, k] & <0 .
\end{aligned}
$$

Proof. If $A$ is t.n.p. the inequality (3.1) holds. Moreover, since $A_{1}$ is a nonsingular t.n.p. matrix, (3.2) and (3.3) follow by [3].

Conversely, by (3.1) for all $\alpha \in \mathcal{Q}_{k, r}$ the following inequality holds

$$
\operatorname{det} A_{1}[\alpha \mid 1,2, \ldots, k] \leq 0 .
$$

Then, by (3.2), (3.3), (3.4) and [3, Theorem 4.1] we have that $A_{1}$ is a nonsingular t.n.p. matrix. As a consequence $A_{1}$ admits a factorization $A_{1}=L_{A_{1}} D_{A_{1}} U_{A_{1}}$, where $L_{A_{1}} \in \mathbb{R}^{r \times r}$ is a unit lower triangular TP matrix, $D_{A_{1}}=\operatorname{diag}\left(-d_{1}, d_{2}, \ldots, d_{r}\right) \in \mathbb{R}^{r \times r}$, with $d_{i}>0, i=1,2, \ldots, r$, and $U_{A_{1}} \in \mathbb{R}^{r \times r}$ is a unit upper triangular TP matrix.

Since $A=F A_{1}$, where $F \in \mathbb{R}^{n \times r}$ is a unit lower reduced echelon matrix we have that

$$
A=F A_{1}=F\left(L_{A_{1}} D_{A_{1}} U_{A_{1}}\right)=\left(F L_{A_{1}}\right) D_{A_{1}} U_{A_{1}}=L D U
$$

where $D=D_{A_{1}}, U=U_{A_{1}}$ and $L=F L_{A_{1}}$ is a unit lower echelon matrix such that

$$
\operatorname{det} A[\alpha \mid 1,2, \ldots, k]=-\prod_{i=1}^{k} d_{i} \operatorname{det} L[\alpha \mid 1,2, \ldots, k] \leq 0 \Longrightarrow L[\alpha \mid 1,2, \ldots, k] \geq 0 .
$$

Hence $L$ is TP by [6, Proposition 2] and we have that $A$ is a t.n.p. matrix by [5]. 
The following proposition allows us to give a characterization by minors of rectangular t.n.p. matrices without full rank.

Proposition 3.2. Let $A=\left(a_{i j}\right) \in \mathbb{R}^{n \times m}$ be a matrix with $\operatorname{rank}(A)=r, a_{n m} \leq 0$ and the remaining entries less than zero. Consider the matrices $A_{1} \in \mathbb{R}^{r \times m}, A_{2} \in$ $\mathbb{R}^{n \times r}, F \in \mathbb{R}^{n \times r}$ in unit lower reduced echelon form and $C \in \mathbb{R}^{r \times m}$ in unit upper reduced echelon form, such that $A=F A_{1}=A_{2} C$. Then, $A$ is t.n.p. if and only if $A_{1}$ and $A_{2}$ are t.n.p.

Proof. Suppose that $A$ is t.n.p. Since $F$ and $C$ are matrices in lower and upper echelon form, respectively, we have that $A_{1}$ and $A_{2}$ are the submatrices of $A$ formed by its first $r$ linearly independent rows or columns, respectively. Then, they are t.n.p. matrices.

Conversely, since $A_{1}$ is t.n.p. with full row rank by [5, Theorem 9], $A_{1}=L_{1} D_{1} U_{1}$ is the unique full rank factorization in unit echelon form, where $L_{1} \in \mathbb{R}^{r \times r}$ is a unit lower triangular TP matrix, $D_{1}=\operatorname{diag}\left(\begin{array}{llll}-d_{11} & d_{12} & \ldots & d_{1 r}\end{array}\right) \in \mathbb{R}^{r \times r}$ with $d_{1 i}>0$ for $i=1,2, \ldots, r, \quad U_{1} \in \mathbb{R}^{r \times m}$ is a unit upper echelon TP matrix and $\operatorname{rank}\left(L_{1}\right)=$ $\operatorname{rank}\left(U_{1}\right)=r$. Then, $A$ admits the following full rank factorization in unit echelon form

$$
A=F A_{1}=F\left(L_{1} D_{1} U_{1}\right)=\left(F L_{1}\right) D_{1} U_{1} .
$$

Analogously, $A_{2}$ is a t.n.p. matrix with full column rank which admits the unique full rank factorization in unit echelon form $A_{2}=L_{2} D_{2} U_{2}$ where $L_{2} \in \mathbb{R}^{n \times r}$ is a unit lower echelon TP matrix, $D_{2}=\operatorname{diag}\left(\begin{array}{llll}-d_{21} & d_{22} & \ldots & d_{2 r}\end{array}\right) \in \mathbb{R}^{r \times r}$ with $d_{2 i}>0$ for $i=1,2, \ldots, r, \quad U_{2} \in \mathbb{R}^{r \times r}$ is a unit upper triangular $\mathrm{TP}$ matrix and $\operatorname{rank}\left(L_{2}\right)=$ $\operatorname{rank}\left(U_{2}\right)=r$. In this case, $A$ admits the full rank factorization in unit echelon form

$$
A=A_{2} C=\left(L_{2} D_{2} U_{2}\right) C=L_{2} D_{2}\left(U_{2} C\right) .
$$

Since the full rank factorization in unit echelon form is unique [4], we have that

$$
A=\left(F L_{1}\right) D_{1} U_{1}=L_{2} D_{2}\left(U_{2} C\right) .
$$

Then $F L_{1}=L_{2}$, which implies that $F L_{1}$ is a unit lower echelon TP matrix. Therefore, by [5, Theorem 9] $A$ is t.n.p. matrix.

Corollary 3.3. Let $A=\left(a_{i j}\right) \in \mathbb{R}^{n \times m}$ be a matrix with $\operatorname{rank}(A)=r, a_{n m} \leq 0$ and the remaining entries less than zero. Suppose that $A=F \bar{A} C$, with $F \in \mathbb{R}^{n \times r}$ unit lower reduced echelon matrix, $\bar{A} \in \mathbb{R}^{r \times r}$ nonsingular matrix and $C \in \mathbb{R}^{r \times m}$ unit upper reduced echelon matrix. Then, $A$ is t.n.p. if and only if $\bar{A} C$ and $F \bar{A}$ are t.n.p.

Proof. Consider the matrices $A_{1}=\bar{A} C$ and $A_{2}=F \bar{A}$. Then $A=F A_{1}$ with $F \in \mathbb{R}^{n \times r}$ unit lower reduced echelon matrix and $A=A_{2} C$ with $C \in \mathbb{R}^{r \times m}$ unit upper reduced echelon matrix. By Proposition 3.2 the result holds. 
The Propositions 3.1 and 3.2 will be used to prove the following characterization by minors of rectangular t.n.p. matrices.

Proposition 3.4. Let $A=\left(a_{i j}\right) \in \mathbb{R}^{n \times m}$ be a matrix with $\operatorname{rank}(A)=r, a_{n m} \leq 0$ and the remaining entries less than zero. Suppose that $A_{1} \in \mathbb{R}^{r \times m}$ is the submatrix of $A$ formed by its first $r$ linearly independent rows, $A_{2} \in \mathbb{R}^{n \times r}$ is the submatrix of $A$ formed by the first $r$ linearly independent columns and $\bar{A} \in \mathbb{R}^{r \times r}$ is the nonsingular submatrix of $A$ formed by its first $r$ linearly independent rows and columns. Then, $A$ is t.n.p. if and only if for each $k=1,2, \ldots, r$, the following inequalities hold

$$
\begin{aligned}
& \operatorname{det} A_{2}[\alpha \mid 1,2, \ldots, k] \leq 0 \quad \forall \alpha \in \mathcal{Q}_{k, n}, \\
& \operatorname{det} A_{1}[1,2, \ldots, k \mid \beta] \leq 0 \quad \forall \beta \in \mathcal{Q}_{k, m}, \\
& \operatorname{det} \bar{A}[1,2, \ldots, k]<0 .
\end{aligned}
$$

Proof. Suppose that $A$ is t.n.p. Since $A_{1}$ and $A_{2}$ are submatrices of $A$, the inequalities (3.5) and (3.6) hold. Moreover, note that $\bar{A}$ is the nonsingular submatrix of $A_{2}\left(A_{1}\right)$ formed by the first $r$ linearly independent rows (columns), then $\bar{A}$ is a nonsingular t.n.p. matrix and by [3, Theorem 4.1] the inequality (3.7) holds.

Conversely, applying Proposition 3.1 to $A_{2} \in \mathbb{R}^{n \times r}$ we know that $A_{2}$ is t.n.p. if and only if for each $k=1,2, \ldots, r$ the following inequalities hold

$$
\begin{aligned}
\operatorname{det} A_{2}[\alpha \mid 1,2, \ldots, k] \leq 0 & \forall \alpha \in \mathcal{Q}_{k, n}, \\
\operatorname{det} \bar{A}[1,2, \ldots, k \mid \beta] \leq 0 & \forall \beta \in \mathcal{Q}_{k, r}, \\
\operatorname{det} \bar{A}[1,2, \ldots, k]<0 . &
\end{aligned}
$$

Now, applying Proposition 3.1 to $A_{1}^{T} \in \mathbb{R}^{m \times r}$ we know that $A_{1}$ is t.n.p. if and only if for each $k=1,2, \ldots, r$ the following inequalities hold

$$
\begin{aligned}
\operatorname{det} A_{1}[1,2, \ldots, k \mid \beta] \leq 0 & \forall \beta \in \mathcal{Q}_{k, m}, \\
\operatorname{det} \bar{A}[\alpha \mid 1,2, \ldots, k] \leq 0 & \forall \alpha \in \mathcal{Q}_{k, r}, \\
\operatorname{det} \bar{A}[1,2, \ldots, k]<0 . &
\end{aligned}
$$

Since the inequalities (3.8) and (3.11) implies the inequalities (3.12) and (3.9) respectively, we can assure that $A_{1}$ and $A_{2}$ are t.n.p. matrices. Then, $A$ is a t.n.p. matrix by Proposition 3.2.

4. A characterization of rectangular t.n.p. matrices by their thin QR factorization. In [11] the authors introduce the concepts of lowerly TP and $\gamma$-matrix for nonsingular TP matrices, which allow to characterize this class of matrices by their QR factorizations. The extension of these concepts and characterization for rectangular TP matrices are given in [6]. In this section we give an equivalent characterization 
for rectangular t.n.p. matrices by using the concepts of lowerly t.n.p. matrix and quasi $\gamma$-matrix. From now on we denote by $I_{(-1)}$ the diagonal matrix $\operatorname{diag}(-1,1, \ldots, 1)$.

Definition 4.1. Consider $A \in \mathbb{R}^{n \times r}$ with $\operatorname{rank}(A)=r$. Then, $\mathrm{A}$ is lowerly t.n.p. if and only if it can be decomposed in the form $A=L D U$, where $L \in \mathbb{R}^{n \times r}$ is a unit lower echelon matrix, $D=\operatorname{diag}\left(-d_{1}, d_{2}, \ldots, d_{r}\right)$ is a nonsingular matrix, $U \in \mathbb{R}^{r \times r}$ is a unit upper triangular matrix and $L D I_{(-1)}$ is a TP matrix.

Note that if $L D I_{(-1)}$ is a TP matrix then, $L$ and $D I_{(-1)}=\operatorname{diag}\left(d_{1}, d_{2}, \ldots, d_{r}\right)$ are TP matrices. The following proposition presents the characterization of lowerly t.n.p. matrices.

Proposition 4.2. Let $A \in \mathbb{R}^{n \times r}$ be a matrix with $\operatorname{rank}(A)=r$. Consider the nonsingular matrix $A_{1} \in \mathbb{R}^{r \times r}$ formed by the first $r$ linearly independent rows of A. Then, $A$ is lowerly t.n.p. if and only if for each $k=1,2, \ldots, r$, the following inequalities hold

$$
\begin{aligned}
\operatorname{det} A[\alpha \mid 1,2, \ldots, k] & \leq 0, \quad \text { for all } \alpha \in \mathcal{Q}_{k, n} \\
\operatorname{det} A_{1}[1,2, \ldots, k] & <0 .
\end{aligned}
$$

Proof. If $A$ is lowerly t.n.p. it can be factorized as $A=L D U$, where $L \in \mathbb{R}^{n \times r}$ is a unit lower echelon matrix, $D=\operatorname{diag}\left(-d_{1}, d_{2}, \ldots, d_{r}\right)$ with $d_{i}>0$ for $i=1,2, \ldots, r$, $U \in \mathbb{R}^{r \times r}$ is a unit upper triangular matrix and $L D I_{(-1)}$ is a TP matrix. Then

$$
\operatorname{det} A[\alpha \mid 1,2, \ldots, k]=-\left(\prod_{i=1}^{k} d_{i}\right) \operatorname{det} L[\alpha \mid 1,2, \ldots, k] \leq 0
$$

Now, suppose that $s_{1}, s_{2}, \ldots, s_{r}$ are the indices of the first $r$ linearly independent rows of $A$. Since $A=L D U$ the submatrix $A_{1}$ can be obtained as follow

$$
A_{1}=L\left[s_{1}, s_{2}, \ldots, s_{r} \mid 1,2, \ldots, r\right] D U
$$

and obviously the inequality (4.2) holds.

Conversely, since $A_{1}$ is the nonsingular submatrix of $A$ formed by the first $r$ linearly independent rows, by (4.2) we know that $A$ admits the factorization $A=$ $L D U$, where $L \in \mathbb{R}^{n \times r}$ is a unit lower echelon matrix, $D=\operatorname{diag}\left(-d_{1}, d_{2}, \ldots, d_{r}\right)$ with $d_{i}>0$ for $i=1,2, \ldots, r$, and $U \in \mathbb{R}^{r \times r}$ is unit upper triangular matrix. Then, by (4.1) we have

$$
\operatorname{det} A[\alpha \mid 1,2, \ldots, k]=-\left(\prod_{i=1}^{k} d_{i}\right) \operatorname{det} L[\alpha \mid 1,2, \ldots, k] \leq 0
$$


which implies that

$$
\operatorname{det} L[\alpha \mid 1,2, \ldots, k] \geq 0
$$

for all $\alpha \in \mathcal{Q}_{k, n}, k=1,2, \ldots, r$. Then, $L$ is TP by [6, Proposition 1]. As a consequence $L D I_{(-1)}$ is a TP matrix because $D I_{(-1)}$ is also TP. Therefore, $A$ is lowerly t.n.p.

Definition 4.3. A matrix $A \in \mathbb{R}^{n \times r}$ with $\operatorname{rank}(A)=r$ is said to be a quasi $\gamma$-matrix if it is lowerly t.n.p. and in the factorization $A=L D U,\left(D U I_{(-1)}\right)^{-1}$ is TP.

EXAMPLE 4.4. The rectangular matrix

$$
A=\left[\begin{array}{rrr}
-1 & -5 & 0 \\
-1 & 1 & -2 \\
-2 & 2 & 1 \\
-3 & 3 & -1
\end{array}\right]
$$

is a quasi $\gamma$-matrix since,

$$
A=\left[\begin{array}{lll}
1 & 0 & 0 \\
1 & 1 & 0 \\
2 & 2 & 1 \\
3 & 3 & 1
\end{array}\right]\left[\begin{array}{rrr}
-1 & 0 & 0 \\
0 & 6 & 0 \\
0 & 0 & 5
\end{array}\right]\left[\begin{array}{ccc}
1 & 5 & 0 \\
0 & 1 & -1 / 3 \\
0 & 0 & 1
\end{array}\right]=L D U
$$

with

$$
L D I_{(-1)}=\left[\begin{array}{ccc}
1 & 0 & 0 \\
1 & 6 & 0 \\
2 & 12 & 5 \\
3 & 18 & 5
\end{array}\right] \text { and }\left(D U I_{(-1)}\right)^{-1}=\left[\begin{array}{ccc}
1 & 5 / 6 & 1 / 3 \\
0 & 1 / 6 & 1 / 15 \\
0 & 0 & 1 / 5
\end{array}\right]
$$

lower and upper triangular TP matrices, respectively.

Remark 4.5. If $A \in \mathbb{R}^{n \times 2}$ with $\operatorname{rank}(A)=2$ is a t.n.p. matrix then it is easy to prove that $A$ is a quasi $\gamma$-matrix. The converse is not true in general, as we can see with the following matrix, which is a quasi $\gamma$-matrix but not a t.n.p. matrix,

$$
A=\left[\begin{array}{rr}
-2 & -2 \\
-1 & 3 \\
-2 & 6
\end{array}\right]=\left[\begin{array}{cc}
1 & 0 \\
1 / 2 & 1 \\
1 & 2
\end{array}\right]\left[\begin{array}{rr}
-2 & 0 \\
0 & 4
\end{array}\right]\left[\begin{array}{ll}
1 & 1 \\
0 & 1
\end{array}\right]
$$

From the quasi $\gamma$-matrices we give a characterization of rectangular t.n.p. matrices by their thin $Q R$ factorization. 
Theorem 4.6. Let $A=\left(a_{i j}\right) \in \mathbb{R}^{n \times r}$ be a matrix with $\operatorname{rank}(A)=r, a_{n r} \leq 0$ and the remaining entries less than zero. Then, $A$ is t.n.p if and only if there exists a quasi $\gamma$-matrix $Q_{1} \in \mathbb{R}^{n \times r}$ with orthonormal columns, an orthogonal quasi $\gamma$-matrix $Q_{2} \in \mathbb{R}^{r \times r}$, a nonsingular upper triangular TP matrix $R_{1} \in \mathbb{R}^{r \times r}$ and an upper echelon TP matrix $R_{2} \in \mathbb{R}^{r \times n}$ such that $A=Q_{1} R_{1}$ and $A^{T}=Q_{2} R_{2}$.

Proof. Suppose that $A$ is t.n.p. with full column rank. By [14] $A$ admits a unique thin $Q R$ factorization $A=Q_{1} R_{1}$, where $Q_{1} \in \mathbb{R}^{n \times r}$ has orthonormal columns and $R_{1}$ is nonsingular upper triangular. Since $A^{T} A$ is a TP matrix such that $A^{T} A=R_{1}^{T} R_{1}$, where $R_{1}$ is its upper triangular Cholesky factor, by [6] $R_{1}$ is a nonsingular upper triangular TP matrix. If we denote by $r_{1_{i}}>0, i=1,2, \ldots, r$, the main diagonal entries of $R_{1}$, we have that

$$
\operatorname{det} A[\alpha \mid 1,2, \ldots, k]=\prod_{i=1}^{k} r_{1_{i}} \operatorname{det} Q_{1}[\alpha \mid 1,2, \ldots, k] \leq 0 .
$$

Then, $\operatorname{det} Q_{1}[\alpha \mid 1,2, \ldots, k] \leq 0$.

Moreover, since $A$ is t.n.p., it admits the full rank factorization in unit echelon form $A=L_{A} D_{A} U_{A}$, where $L_{A} \in \mathbb{R}^{n \times r}$ is a unit lower echelon TP matrix, $D_{A}=$ $\operatorname{diag}\left(-d_{1}, d_{2}, \ldots, d_{r}\right)$ with $d_{i}>0$ for $i=1,2, \ldots, r$, and $U_{A} \in \mathbb{R}^{r \times r}$ is a unit upper triangular TP matrix. If we represent by $D_{R_{1}}=\operatorname{diag}\left(r_{1_{1}}, r_{1_{2}}, \ldots, r_{1_{r}}\right)$, it follows that

$$
\begin{aligned}
A=Q_{1} R_{1}=L_{A} D_{A} U_{A} & \Longrightarrow Q_{1}=L_{A} D_{A} U_{A} R_{1}^{-1} \\
& =L_{A}\left(D_{A} D_{R_{1}}^{-1}\right)\left(D_{R_{1}} U_{A} R_{1}^{-1}\right)=L_{Q_{1}} D_{Q_{1}} U_{Q_{1}},
\end{aligned}
$$

where $L_{Q_{1}}=L_{A} \in \mathbb{R}^{n \times r}$ is a unit lower echelon TP matrix, $D_{Q_{1}}=D_{A} D_{R_{1}}^{-1}=$ $\operatorname{diag}\left(-d_{q_{1}}, d_{q_{2}}, \ldots, d_{q_{r}}\right)$, with $d_{q_{i}}=d_{i} / r_{1_{i}}>0$ for $i=1,2, \ldots, r$, and $U_{Q_{1}}=$ $D_{R_{1}} U_{A} R_{1}^{-1} \in \mathbb{R}^{r \times r}$ is a unit upper triangular matrix.

Since $L_{Q_{1}}$ and $D_{Q_{1}} I_{(-1)}$ are TP, their product is also TP, which implies that $Q_{1}$ is lowerly t.n.p.

On the other hand,

$$
\begin{aligned}
Q_{1}^{T}=U_{Q_{1}}^{T} D_{Q_{1}} L_{Q_{1}}^{T} & \Longrightarrow\left(U_{Q_{1}}^{T} D_{Q_{1}}\right)^{-1} Q_{1}^{T}=L_{Q_{1}}^{T} \\
& \Longrightarrow\left(U_{Q_{1}}^{T} D_{Q_{1}}\right)^{-1}=L_{Q_{1}}^{T} Q_{1} \\
& \Longrightarrow\left(U_{Q_{1}}^{T} D_{Q_{1}}\right)^{-1} I_{(-1)}=L_{Q_{1}}^{T} Q_{1} I_{(-1)} .
\end{aligned}
$$

Hence by the Binet-Cauchy identity [1],

$$
\begin{gathered}
\operatorname{det}\left(U_{Q_{1}}^{T} D_{Q_{1}}\right)^{-1} I_{(-1)}[\alpha \mid 1,2, \ldots, k]=\operatorname{det}\left(L_{Q_{1}}^{T} Q_{1} I_{(-1)}\right)[\alpha \mid 1,2, \ldots, k]= \\
=-\sum_{\forall \gamma \in \mathcal{Q}_{k, r}} \operatorname{det}\left(L_{Q_{1}}^{T}\right)[\alpha \mid \gamma] \operatorname{det} Q_{1}[\gamma \mid 1,2, \ldots, k] \geq 0
\end{gathered}
$$


Therefore by [1, Corollary 2.2], the matrix

$$
\left(U_{Q_{1}}^{T} D_{Q_{1}}\right)^{-1} I_{(-1)}=D_{Q_{1}}^{-T} U_{Q_{1}}^{-T} I_{(-1)}^{-T}=\left(I_{(-1)}^{-1} U_{Q_{1}}^{-1} D_{Q_{1}}^{-1}\right)^{T}
$$

is lower triangular TP. Consequently, $\left(D_{Q_{1}} U_{Q_{1}} I_{(-1)}\right)^{-1}$ is an upper triangular TP matrix, which implies that $Q_{1}$ is a quasi $\gamma$-matrix.

Since $A^{T} \in \mathbb{R}^{r \times n}$ is also a t.n.p. matrix, as in the previous case

$$
A^{T}=Q_{2} R_{2},
$$

where $Q_{2} \in \mathbb{R}^{r \times r}$ is an orthogonal quasi $\gamma$-matrix and $R_{2} \in \mathbb{R}^{r \times n}$ is an upper echelon TP matrix.

Conversely, since $Q_{1}$ is a lowerly t.n.p. matrix, by Proposition 4.2 for all $k=$ $1,2, \ldots, r$, the following inequalities hold

$$
\begin{aligned}
\operatorname{det} Q_{1}[\alpha \mid 1,2, \ldots, k] & \leq 0, \quad \text { for all } \alpha \in \mathcal{Q}_{k, n} \\
\operatorname{det} Q_{1_{1}}[1,2, \ldots, k] & <0 .
\end{aligned}
$$

where the columns of $Q_{1_{1}}$ are the first $r$ linearly independent rows of $Q_{1}$. Then, from (4.3) we have that

$$
\operatorname{det} A[\alpha \mid 1,2, \ldots, k] \leq 0, \quad \text { for all } \alpha \in \mathcal{Q}_{k, n}, \quad k=1,2, \ldots, r .
$$

On the other hand, since the submatrix of $A$ formed by its first $r$ linearly independent rows can be obtained as $A_{1}=Q_{1_{1}} R_{1}$, it follows by (4.4) that

$$
\operatorname{det} A_{1}[1,2, \ldots, k]<0, \quad k=1,2, \ldots, r
$$

We also have that $A_{1}^{T}=Q_{2} \tilde{R}_{2}$, where $\tilde{R}_{2}$ is the upper triangular submatrix of $R_{2}$ formed by its first $r$ linearly independent columns. Moreover, since $Q_{2}$ is a lowerly t.n.p. matrix

$$
\operatorname{det} A_{1}^{T}[\beta \mid 1,2, \ldots, k]=\left(\prod_{i=1}^{k} \tilde{r}_{2_{i}}\right) \operatorname{det} Q_{2}[\beta \mid 1,2, \ldots, k] \leq 0,
$$

where $\tilde{r}_{2_{i}}$, with $i=1,2, \ldots, r$, are main diagonal of $\tilde{R}_{2}$. Therefore,

$$
\operatorname{det} A_{1}[1,2, \ldots, k \mid \beta] \leq 0, \quad \text { for all } \alpha \in \mathcal{Q}_{k, n}, \quad k=1,2, \ldots, r .
$$

Finally, $A$ is a t.n.p. matrix by Proposition 3.1.

Next theorem gives an extension of Theorem 4.6 to rectangular matrices without full column rank. 
Theorem 4.7. Let $A=\left(a_{i j}\right) \in \mathbb{R}^{n \times m}$ be a matrix with $\operatorname{rank}(A)=r, a_{n m} \leq 0$ and the remaining entries less than zero. Then, $A$ is t.n.p. if and only if there exist two quasi $\gamma$-matrices $Q_{1} \in \mathbb{R}^{n \times r}$ and $Q_{2} \in \mathbb{R}^{m \times r}$, both with orthonormal columns and two upper echelon TP matrices $R_{1} \in \mathbb{R}^{r \times m}$ and $R_{2} \in \mathbb{R}^{r \times n}$, such that $A=Q_{1} R_{1}$ and $A^{T}=Q_{2} R_{2}$.

Proof. Suppose that $A$ is t.n.p. Since $\operatorname{rank}(A)=r$, then $A=\tilde{A} C_{1}$, where $\tilde{A} \in \mathbb{R}^{n \times r}$ is a t.n.p. matrix formed by the first $r$ linearly independent columns of $A$ and $C_{1} \in \mathbb{R}^{r \times m}$ is a unit upper reduced matrix. Applying Theorem 4.6 to $\tilde{A}$ we have that

$$
\tilde{A}=\tilde{Q}_{1} \tilde{R}_{1},
$$

where $\tilde{Q}_{1} \in \mathbb{R}^{n \times r}$ is a quasi $\gamma$-matrix with orthonormal columns and $\tilde{R}_{1} \in \mathbb{R}^{r \times r}$ is a nonsingular upper triangular TP matrix. In consequence,

$$
A=\tilde{A} C_{1}=\tilde{Q}_{1} \tilde{R}_{1} C_{1}=\tilde{Q}_{1}\left(\tilde{R}_{1} C_{1}\right)=Q_{1} R_{1}
$$

where $Q_{1}=\tilde{Q}_{1} \in \mathbb{R}^{n \times r}$ is a quasi $\gamma$-matrix with orthonormal columns and $R_{1}=$ $\tilde{R}_{1} C_{1} \in \mathbb{R}^{r \times m}$ is an upper echelon matrix. Since $R_{1}^{T}=A^{T} Q_{1}$,

$$
\operatorname{det} R_{1}^{T}[\alpha \mid 1,2, \ldots, k]=\sum_{\forall \gamma \in \mathcal{Q}_{k, r}} \operatorname{det} A^{T}[\alpha \mid \gamma] \operatorname{det} Q_{1}[\gamma \mid 1,2, \ldots, k] \geq 0
$$

and $R_{1}^{T}$ is a lower echelon TP matrix by [6, Proposition 1]. Therefore $R_{1}$ is an upper echelon TP matrix.

Analogously, since $A^{T} \in \mathbb{R}^{m \times n}$ is a t.n.p. matrix with $\operatorname{rank}\left(A^{T}\right)=r$,

$$
A^{T}=\tilde{A}^{T} C_{2},
$$

where $\tilde{A}^{T} \in \mathbb{R}^{m \times r}$ is a t.n.p. matrix formed by the first $r$ linearly independent columns of $A^{T}$ and $C_{2} \in \mathbb{R}^{r \times n}$ is a unit upper reduced matrix. Reasoning in the same way we obtain that $A^{T}=Q_{2} R_{2}$, where $Q_{2} \in \mathbb{R}^{m \times r}$ is a quasi $\gamma$-matrix with orthonormal columns and $R_{2} \in \mathbb{R}^{r \times n}$ is an upper echelon TP matrix.

Conversely, suppose that $A=Q_{1} R_{1}$. Since $Q_{1} \in \mathbb{R}^{n \times r}$ is a quasi $\gamma$-matrix with $\operatorname{rank}\left(Q_{1}\right)=r$, it admits the full rank factorization

$$
Q_{1}=L_{Q_{1}} D_{Q_{1}} U_{Q_{1}}
$$

where $L_{Q_{1}} \in \mathbb{R}^{n \times r}$ is a unit lower echelon matrix, $D_{Q_{1}}=\operatorname{diag}\left(-d_{q_{1_{1}}}, d_{q_{1_{2}}}, \ldots, d_{q_{1_{r}}}\right)$ with $d_{q_{1_{i}}}>0$ for $i=1,2, \ldots, r, U_{Q_{1}} \in \mathbb{R}^{r \times r}$ is a unit upper triangular matrix, such that $\operatorname{rank}\left(L_{Q_{1}}\right)=\operatorname{rank}\left(U_{Q_{1}}\right)=r$ and $L_{Q_{1}} D_{Q_{1}} I_{(-1)}$ ia a TP matrix (with $L_{Q_{1}}$ also a TP matrix). Then,

$$
\begin{aligned}
A & =\left(L_{Q_{1}} D_{Q_{1}} U_{Q_{1}}\right) R_{1}=\left(L_{Q_{1}} D_{Q_{1}}\right)\left(U_{Q_{1}} R_{1}\right) \\
& =\left(L_{Q_{1}} D_{Q_{1}}\right)\left(D_{1} U_{1}\right)=L_{Q_{1}}\left(D_{Q_{1}} D_{1}\right) U_{1}=L_{A} D_{A} U_{A}
\end{aligned}
$$


is a full rank factorization of $A$, with $L_{A}=L_{Q_{1}} \in \mathbb{R}^{n \times r}$ a unit lower echelon TP matrix, $D_{A}=D_{Q_{1}} D_{1}=\operatorname{diag}\left(-d_{1_{1}}, d_{1_{2}}, \ldots, d_{1_{r}}\right)$ with $d_{1_{i}}>0$ for $i=1,2, \ldots, r$, $U_{A}=U_{1} \in \mathbb{R}^{r \times m}$ unit upper echelon matrix and $\operatorname{rank}\left(L_{A}\right)=\operatorname{rank}\left(U_{A}\right)=r$.

Analogously, $A^{T}=Q_{2} R_{2}$ where $Q_{2} \in \mathbb{R}^{m \times r}$ is a $\gamma$-matrix with $\operatorname{rank}\left(Q_{2}\right)=r$. Then, $Q_{2}$ admits the following full rank factorization

$$
Q_{2}=L_{Q_{2}} D_{Q_{2}} U_{Q_{2}}
$$

where $L_{Q_{2}} \in \mathbb{R}^{m \times r}$ is a unit lower echelon matrix, $D_{Q_{2}}=\operatorname{diag}\left(-d_{q_{2_{1}}}, d_{q_{2_{2}}}, \ldots, d_{q_{2_{r}}}\right)$ with $d_{q_{2_{i}}}>0$ for $i=1,2, \ldots, r, U_{Q_{2}} \in \mathbb{R}^{r \times r}$ is a unit upper triangular matrix, such that $\operatorname{rank}\left(L_{Q_{2}}\right)=\operatorname{rank}\left(U_{Q_{2}}\right)=r$, and $L_{Q_{1}} D_{Q_{1}} I_{(-1)}$ is a TP matrix (with $L_{Q_{2}}$ also a TP matrix). As a consequence,

$$
\begin{aligned}
A^{T} & =\left(L_{Q_{2}} D_{Q_{2}} U_{Q_{2}}\right) R_{2}=\left(L_{Q_{2}} D_{Q_{2}}\right)\left(U_{Q_{2}} R_{2}\right) \\
& =\left(L_{Q_{2}} D_{Q_{2}}\right)\left(D_{2} U_{2}\right)=L_{Q_{2}}\left(D_{Q_{2}} D_{2}\right) U_{2}=L_{A^{T}} D_{A^{T}} U_{A^{T}}
\end{aligned}
$$

with $L_{A^{T}}=L_{Q_{2}} \in \mathbb{R}^{m \times r}$ unit lower echelon TP matrix, $U_{A^{T}}=U_{2} \in \mathbb{R}^{r \times n}$ unit upper echelon matrix with $\operatorname{rank}\left(L_{A^{T}}\right)=\operatorname{rank}\left(U_{A^{T}}\right)=r$ and $D_{A^{T}}=D_{Q_{2}} D_{2}=$ $\operatorname{diag}\left(-d_{2_{1}}, d_{2_{2}}, \ldots, d_{2_{r}}\right)$ with $d_{2_{i}}>0$, for $i=1,2, \ldots, r$.

Therefore,

$$
A=L_{A} D_{A} U_{A}=\left(L_{A^{T}} D_{A^{T}} U_{A^{T}}\right)=U_{A^{T}}^{T} D_{A^{T}} L_{A^{T}}^{T},
$$

where $U_{A}=L_{A^{T}}^{T} \in \mathbb{R}^{r \times m}$ is a unit upper echelon TP matrix because the full rank factorization in echelon form of any matrix is unique [4]. Finally, $A$ is a t.n.p. matrix by $[5$, Theorem 8$]$.

5. A characterization of rectangular t.n. matrices by their thin QR factorization. In this section we characterize the rectangular t.n. matrices by their thin $Q R$ factorization. In [6, Theorem 6] the authors give the following characterization of rectangular t.n. matrices

Theorem 5.1. Consider $A=\left(a_{i j}\right) \in \mathbb{R}^{n \times m}$ with $a_{n m}<0$ and $n \leq m$. A is t.n. if and only if for each $k=1,2, \ldots, n$, the following inequalities hold

$$
\begin{aligned}
& \operatorname{det} A[\alpha \mid\{1,2, \ldots, k\}]<0, \quad \text { for all } \alpha \in \mathcal{Q}_{k, n}^{0} \\
& \operatorname{det} A[\{1,2, \ldots, k\} \mid \beta]<0, \quad \text { for all } \beta \in \mathcal{Q}_{k, m}^{0}
\end{aligned}
$$

In analogous way to the previous section we extend to rectangular matrices the concept of lowerly t.n. matrix and give the characterization of this class of matrices by minors. 
Definition 5.2. Consider $A \in \mathbb{R}^{n \times r}$ with $\operatorname{rank}(A)=r$. Then, A is lowerly t.n. if and only if it can be decomposed in the form $A=L D U$, where $L \in \mathbb{R}^{n \times r}$ is a unit lower echelon matrix, $D=\operatorname{diag}\left(-d_{1}, d_{2}, \ldots, d_{r}\right)$ is a nonsingular matrix, $U \in \mathbb{R}^{r \times r}$ is a unit upper triangular matrix and $L D I_{(-1)}$ is a $\Delta \mathrm{STP}$ matrix.

Note that if $L D I_{(-1)}$ is a $\Delta \mathrm{STP}$ matrix then, $L$ is a $\Delta \mathrm{STP}$ matrix and $D I_{(-1)}=$ $\operatorname{diag}\left(d_{1}, d_{2}, \ldots, d_{r}\right)$ is a TP matrix. The following proposition presents the characterization of lowerly quasi STP matrices.

Proposition 5.3. A matrix $A=\left(a_{i j}\right) \in \mathbb{R}^{n \times m}$ with $\operatorname{rank}(A)=m$ and $a_{n m}<0$, is lowerly t.n. if and only if for each $k=1,2, \ldots, m$ the following inequalities hold

$$
\operatorname{det} A[\alpha \mid 1,2, \ldots, k]<0 \quad \text { for all } \alpha \in \mathcal{Q}_{k, n}^{0} .
$$

Proof. If $A$ is lowerly t.n. then $A=L D U$, with $D=\operatorname{diag}\left(-d_{1}, d_{2}, \ldots, d_{m}\right)$, $d_{i}>0, i=1,2, \ldots, m$. By $[6$, Remark 3] we have that

$$
\begin{aligned}
\operatorname{det} A[\alpha \mid 1,2, \ldots, k] & =\operatorname{det}(L D U)[\alpha \mid 1,2, \ldots, k]=\operatorname{det}(L D)[\alpha \mid 1,2, \ldots, k] \\
& =\left(-\prod_{i=1}^{k} d_{i}\right) \operatorname{det} L[\alpha \mid 1,2, \ldots, k]<0 \quad \forall \alpha \in \mathcal{Q}_{k, n}^{0} .
\end{aligned}
$$

Conversely, from (5.3) we have that $A$ admits a factorization $A=L D U$ with no pivoting such that $L \in \mathbb{R}^{n \times m}$ is a unit lower echelon matrix, $D=\operatorname{diag}\left(-d_{1}, d_{2}, \ldots, d_{m}\right)$ with $d_{i}>0$, for $i=1,2, \ldots, m$, and $U \in \mathbb{R}^{m \times m}$ is a unit upper triangular matrix. Since for all $\alpha \in \mathcal{Q}_{k, n}^{0}$ it is satisfied that

$$
\operatorname{det} A[\alpha \mid 1,2, \ldots, k]=\left(-\prod_{i=1}^{k} d_{i}\right) \operatorname{det} L[\alpha \mid 1,2, \ldots, k]<0,
$$

then $L$ is $\Delta \mathrm{STP}$ by $[6$, Remark 3], therefore $L D$ is also $\Delta \mathrm{STP}$ and $A$ is lowerly t.n.

Now, the following definition extends to rectangular matrices with full column rank the concept of quasi strict $\gamma$-matrix.

Definition 5.4. A matrix $A \in \mathbb{R}^{n \times r}$, with $\operatorname{rank}(A)=r$, is said to be a quasi strict $\gamma$-matrix if it is lowerly t.n. and in the factorization $A=L D U,\left(D U I_{(-1)}\right)^{-1}$ is $\triangle \mathrm{STP}$.

Next result gives a characterization of the t.n. matrices by their thin $Q R$ factorization using quasi strict $\gamma$-matrices. The proof is similar to Theorem 4.6.

Theorem 5.5. Let $A=\left(a_{i j}\right) \in \mathbb{R}^{n \times m}$ be a matrix with $\operatorname{rank}(A)=m$ and all its entries less than zero. Then, $A$ is t.n. if and only if there exist a quasi strict 
$\gamma$-matrix $Q_{1} \in \mathbb{R}^{n \times m}$ with orthonormal columns and an orthogonal quasi strict $\gamma$ -

matrix $Q_{2} \in \mathbb{R}^{m \times m}$, a nonsingular upper triangular $\Delta S T P$ matrix $R_{1} \in \mathbb{R}^{m \times m}$ and an upper echelon $\triangle S T P$ matrix $R_{2} \in \mathbb{R}^{m \times n}$, such that $A=Q_{1} R_{1}$ and $A^{T}=Q_{2} R_{2}$.

\section{REFERENCES}

[1] T. Ando. Totally positive matrices. Linear Algebra and its Applications, 90:165-219, 1987.

[2] R. B. Bapat and T. E. S. Raghavan. Nonnegative Matrices and Applications. Cambridge University Press, New York, 1997.

[3] R. Cantó, P. Koev, B. Ricarte, and A. M. Urbano. LDU-factorization of Nonsingular Totally Nonpositive Matrices. SIAM Journal on Matrix Analysis and Applications, 30(2):777-782, 2008 .

[4] R. Cantó, B. Ricarte, and A. M. Urbano. Full rank factorization and Flanders theorem. Electronic Journal of Linear Algebra, 18:352-363, 2009.

[5] R. Cantó, B. Ricarte, and A. M. Urbano. Full rank factorization in echelon form of totally nonpositive (negative) rectangular matrices. Linear Algebra and its Applications, 431:22132227, 2009.

[6] R. Cantó, B. Ricarte, and A. M. Urbano. Characterizations of rectangular totally and strictly totally positive matrices. Linear Algebra and its Applications, 432:2623-2633, 2010.

[7] C. W. Cryer. The LU-factorization of Totally Positive Matrices. Linear Algebra and its Applications, 7:83-92, 1973.

[8] S. M. Fallat, A. Herman, M.I. Gekhtman, and C.R. Johnson. Comprension of totally positive matrices. SIAM Journal on Matrix Analysis and Applications, 28:68-80, 2006

[9] S. M. Fallat and P. Van Den Driessche. On matrices with all minors negative. Electronic Journal of Linear Algebra, 7:92-99, 2000.

[10] M. Gasca and C.A. Micchelli. Total Positivity and Applications. Mathematics and its Applications, vol. 359, Kluwer Academic Publishers., Dordrecht, The Netherlands, 1996.

[11] M. Gasca and J. M. Peña. Total positivity, QR factorization and Neville elimination. SIAM Journal on Matrix Analysis and Applications, 4:1132-1140, 1993.

[12] M. Gasca and J. M. Peña. A test for strict sign-regularity. Linear Algebra and its Applications, 197/198:133-142, 1994.

[13] M. Gasca and J. M. Peña. On factorization of totally positive matrices. Mathematics and its Applications, vol. 359, Kluwer Academic Publishers., Dordrecht, The Netherlands, pp. 109-130, 1996.

[14] J. H. Golub and C. F. Van Loan. Matrix Computations. The John Hopkins Univ. Press, 1966.

[15] M. Gassó and J.R. Torregrosa. A totally positive factorization of rectangular matrices by the Neville elimination. SIAM Journal on Matrix Analysis and Applications, 25:986-994, 2004.

[16] T. Parthasarathy. N-matrices. Linear Algebra and its Applications, 139:89-102, 1990.

[17] R. Saigal. On the class of complementary cones and Lemke's algorithm. SIAM Journal on Applied Mathematics, 23:46-60, 1972. 